\title{
Rolling Bearing Fault Signal Extraction Based on Stochastic Resonance-Based Denoising and VMD
}

\author{
Xiaojiao $\mathrm{Gu}^{1}$ and Changzheng Chen ${ }^{1,2}$ \\ ${ }^{1}$ School of Mechanical Engineering, Shenyang University of Technology, Shenyang 110870, China \\ ${ }^{2}$ Liaoning Engineering Center for Vibration and Noise Control, Shenyang 110870, China \\ Correspondence should be addressed to Changzheng Chen; chencz6699@sina.com
}

Received 6 April 2017; Revised 10 August 2017; Accepted 27 August 2017; Published 1 November 2017

Academic Editor: Hyeong Joon Ahn

Copyright ( 2017 Xiaojiao Gu and Changzheng Chen. This is an open access article distributed under the Creative Commons Attribution License, which permits unrestricted use, distribution, and reproduction in any medium, provided the original work is properly cited.

\begin{abstract}
Aiming at the difficulty of early fault vibration signal extraction of rolling bearing, a method of fault weak signal extraction based on variational mode decomposition (VMD) and quantum particle swarm optimization adaptive stochastic resonance (QPSO-SR) for denoising is proposed. Firstly, stochastic resonance parameters are optimized adaptively by using quantum particle swarm optimization algorithm according to the characteristics of the original fault vibration signal. The best stochastic resonance system parameters are output when the signal to noise ratio reaches the maximum value. Secondly, the original signal is processed by optimal stochastic resonance system for denoising. The influence of the noise interference and the impact component on the results is weakened. The amplitude of the fault signal is enhanced. Then the VMD method is used to decompose the denoised signal to realize the extraction of fault weak signals. The proposed method was applied in simulated fault signals and actual fault signals. The results show that the proposed method can reduce the effect of noise and improve the computational accuracy of VMD in noise background. It makes VMD more effective in the field of fault diagnosis. The proposed method is helpful to realize the accurate diagnosis of rolling bearing early fault.
\end{abstract}

\section{Introduction}

Rolling bearing is one of the most critical and easily damaged components in rotating machinery. Its running state is directly related to the performance of the whole mechanical system [1-3]. Therefore, it is significant to implement fault diagnosis for rolling bearings so as to prevent fatal malfunction of rotating machinery [4]. Vibration analysis is one of the most frequently used methods for health monitoring and fault diagnosis of rotating machinery. The vibration signal of mechanical equipment is rich in status information [5, 6]. When a rotating machine has incipient faults or works in a wicked environment, the useful fault signals are often submerged in strong background noise. It will seriously affect the accuracy of fault diagnosis. Therefore, the extraction of weak fault signal is a research hotspot in the field of signal processing [7].

In order to extract useful signals from complex original signals, many scholars have proposed a lot of effective weak signal extraction methods. One category is to extract weak signal from the perspective of denoising, such as wavelet transform, chaos theory, empirical mode decomposition (EMD), local mean decomposition (LMD), ensemble empirical mode decomposition (EEMD), and singular value decomposition (SVD). Under the influence of sampling frequency, the EEMD decomposition error is large. Meng and Xiang [8] proposed an improved EEMD which extracts the real intrinsic mode functions (IMF) by using the correlation coefficients between the original signal and IMFs. With this method, the pseudo low-frequency IMFs can be eliminated. Yi et al. [9] proposed the augmented quaternion singular spectrum analysis multichannel denoising method. This method has a better ability than multivariate EMD method in multisignal processing. Xie et al. [10] proposed an improved LMD based on extension characteristic wave method to eliminate the end effect. Yi et al. [11] proposed a convex optimization algorithm using nonconvex penalty functions based on SVD for extracting weak fault characteristics. Variational mode 
decomposition (VMD) is a new adaptive signal processing method proposed by Dragomiretskiy and Zosso in 2014 [12]. In this method, the frequency center and bandwidth of each component are determined by iteration searching for the optimal solution of the variational mode during the process of obtaining decomposition components. Therefore, the effective separation of the frequency domain can be realized adaptively. The existing study shows that in complicated signal decomposition the VMD has more advantage than EMD [13]. Compared with the recursive filtering mode of EEMD and LMD, VMD is a nonrecursive and variational mode decomposition. It has a solid theoretical basis. Its essence is a number of adaptive Wiener filter groups, which show better noise robustness. Through the reasonable control for the convergence condition, the influence of sampling on VMD is much smaller than EEMD and LMD. In terms of mode separation, the VMD can successfully separate two pure harmonic signals with similar frequencies. At present, the research results of the variational mode decomposition in the field of mechanical fault diagnosis show that VMD has better performance than LMD and EEMD in many aspects [14]. The fault feature extraction based on variational mode decomposition has important application value.

The other category is to use the noise to enhance the fault signal by means of stochastic resonance (SR). Because of its obvious advantages in weak signal detection, SR has become a hotspot in the field of signal processing in recent years $[15,16]$. Han et al. [17] combined wavelet transform with stochastic resonance theory, which can detect several high frequency weak signals in strong noise background by adjusting the amplitude of wavelet multiscale decomposition. Rother et al. [18] combined stochastic resonance with EMD, which is applied to detect deviations in strip travel of hot strip mill. The results show that this method can reduce downtimes and extend the lifetime of components. Barbini et al. [19] focused on the use of SR in a two-dimensional system of gradient type for detection of weak signals submerged in Gaussian noise. The two-dimensional system is more accurate and more reliable than the traditional one-dimensional system. Castiglione et al. [20] used bistable stochastic resonance and Woods-Saxon stochastic resonance in bearing faults detection, respectively. Experimental data shows that each SR method has its own characteristic features. Gao et al. [21] constructed corresponding Duffing oscillator based on chaos theory to monitor weak signal, which reduces the SNR threshold compared with the traditional monitoring method. Lu et al. [22] enhanced stochastic resonance by full-wave signal construction, which surpass the traditional stochastic resonance in the field of fault signal processing and can be used in areas related to weak signal detection. Lin et al. [23] presented a new additive noise driving and aperiodic chirp signal stochastic dynamical system, which can improve the system performance of localization and moving target detection. Breen et al. [24] used the mechanical stochastic resonance to detect weak signals, which harvest weak periodic signals via the movement of an inverted pendulum. Shi et al. [25] combined analytical mode decomposition (AMD) and EEMD with adaptive stochastic resonance, which can improve the decomposition results of EEMD. This method has a good effect in the rolling bearing fault diagnosis. Xiang and Zhong [26] presented a two-step method of Duffing oscillator and stochastic resonance. And this method combined stochastic resonance with the artificial bee colony algorithm. It is favorable to detect weak signal from a strong noise environment. Liu et al. [27] combined wavelet reconstruction and stochastic resonance. The reconstruction scale was selected by comparing the wavelet entropy of each decomposition scale. This fault detection method is more accurate than the conventional wavelet reconstruction. The above studies which combine the stochastic resonance with the signal processing method or the optimization method show that the appropriate application of the stochastic resonance method can make the signal processing method have a better effect in fault diagnosis. Stochastic resonance has important application value in fault diagnosis under strong noise background.

Combining the respective advantages of VMD and SR, this study presents a weak signal extraction method of rolling bearing fault based on VMD and quantum particle swarm optimization (QPSO) adaptive stochastic resonance. First, the parameters of stochastic resonance system are optimized according to the original signal feature and quantum particle swarm optimization algorithm. Thereafter, the original signal is processed by the stochastic resonance so that the noise frequency component is weakened and the fault frequency component is enhanced. Lastly, the signal which is processed by stochastic resonance is decomposed by VMD method. The useful signal features are extracted. The rolling bearing measured signal analysis shows that the proposed method can discharge most of the interfering signal generated by the background noise so that the components obtained by the VMD can better reflect the fault signal information and enhance the useful signal amplitude. The detection results are more accurate and reliable.

The rest of this paper is organized as follows. Section 2 provides a brief description of the principal theory of VMD, SR, and QPSO. Section 3 introduces the proposed fault diagnosis process and discusses the simulation study to validate the performance of the proposed method. Section 4 discusses the case study to validate the practical application value of this method. Lastly, Section 5 presents the conclusion.

\section{Basic Theory}

2.1. Variational Mode Decomposition. VMD algorithm is a new nonrecursive variational mode signal decomposition method, which can decompose the complex input signal into a set of discrete mode components [28]. The implementation steps of the VMD algorithm are as follows.

Step 1. The Hilbert transform is performed for each mode function $u_{k}$ to obtain its analytical signal, as follows:

$$
\left[\delta(t)+\frac{j}{\pi t}\right] * u_{k}(t) .
$$


Step 2. The estimated center frequency $\mathrm{e}^{j \omega_{k} t}$ of $u_{k}$ is mixed. The spectrum of each mode is modulated to the corresponding base band, as follows:

$$
\left[\left(\delta(t)+\frac{j}{\pi t}\right) * u_{k}(t)\right] * \mathrm{e}^{-j \omega_{k} t}
$$

Step 3. Calculate the gradient square $L^{2}$ norm of the above demodulated signal and estimate the bandwidth of each mode signal. The variational problem is constructed as follows:

$$
\begin{aligned}
& \min _{\left\{u_{k}\right\},\left\{\omega_{k}\right\}}\left\{\sum_{k}\left\|\partial_{t}\left[\left(\delta(t)+\frac{j}{\pi t}\right) * u_{k}(t)\right] * \mathrm{e}^{-j \omega_{k} t}\right\|^{2}\right\}, \\
& \text { s.t. } \quad \sum_{k} u_{k}=f,
\end{aligned}
$$

where $\left\{u_{k}\right\}:=\left\{u_{1}, \ldots, u_{k}\right\}$ represents $K$ variational mode components. $\left\{\omega_{k}\right\}:=\left\{\omega_{1}, \ldots, \omega_{k}\right\}$ represents the center frequency of each component. $K$ is the number of variational mode components. $t$ represents the time. $\partial_{t}$ represents the partial derivative of $t . \delta(t)$ represents the impulse function. $j$ is the square root of -1 . $\omega$ represents the circular frequency. $f$ represents the input signal.

Step 4. In order to transform the constraint variational problem into the unconstrained problem, the quadratic penalty factor $\alpha$ is introduced to ensure the accuracy of signal reconstruction in noise condition. The Lagrangian multiplier $\lambda$ is introduced to ensure the stringency of the constraint condition. Through introducing the two parameters, the extended Lagrangian expression is obtained as follows:

$$
\begin{aligned}
& L\left(\left\{u_{k}\right\},\left\{\omega_{k}\right\}, \lambda\right) \\
& :=\alpha \sum_{k}\left\|\partial_{t}\left[\left(\delta(t)+\frac{j}{\pi t}\right) * u_{k}(t)\right] * \mathrm{e}^{-j \omega_{k} t}\right\|_{2}^{2} \\
& +\left\|f(t)-\sum_{k} u_{k}(t)\right\|_{2}^{2} \\
& +\left\langle\lambda(t), f(t)-\sum_{k} u_{k}(t)\right\rangle .
\end{aligned}
$$

Step 5. Solve the extended Lagrangian expression by using the alternate direction method of multipliers (ADMM). Specific steps are as follows:

(1) Initialize $\left\{\widehat{u}_{k}^{1}\right\},\left\{\omega_{k}^{1}\right\}, \widehat{\lambda}^{1}, n$.

(2) Repeat $n=n+1$.

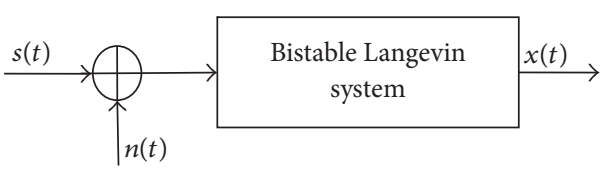

FIGURE 1: SR structure for the bistable Langevin system.

(3) Update $u_{k}, \omega_{k}$, and $\lambda$, and do

$$
\begin{aligned}
\widehat{u}_{k}^{n+1}(\omega) & \longleftarrow \frac{\widehat{f}(\omega)-\sum_{i \neq k} \widehat{u}_{i}(\omega)+\hat{\lambda}(\omega) / 2}{1+2 \alpha\left(\omega-\omega_{k}\right)^{2}}, \\
\omega_{k}^{n+1} & \longleftarrow \frac{\int_{0}^{\infty} \omega\left|\widehat{u}_{k}(\omega)\right|^{2} \mathrm{~d} \omega}{\int_{0}^{\infty}\left|\widehat{u}_{k}(\omega)\right|^{2} \mathrm{~d} \omega}, \\
\hat{\lambda}^{n+1}(\omega) & \longleftarrow \widehat{\lambda}^{n}(\omega)+\tau\left[\widehat{f}(\omega)-\sum_{k} \widehat{u}_{k}^{n+1}(\omega)\right] .
\end{aligned}
$$

(4) Judge whether or not the following conditions are met. If met, the iteration is stopped; otherwise, return to execute Step (2).

$$
\frac{\sum_{k}\left\|\widehat{u}_{k}^{n+1}-\widehat{u}_{k}^{n}\right\|_{2}^{2}}{\left\|\hat{u}_{k}^{n}\right\|_{2}^{2}}<e,
$$

where $e$ is the convergence stopped condition. $k$ is the iteration number.

2.2. Stochastic Resonance. Benzi et al. [29] first introduced the mechanism of stochastic resonance in 1981 and used SR in climatic change. In the field of signal processing, stochastic resonance can transfer the energy of some noise signals to useful signals, which is beneficial to the identification of weak signals submerged in noise [30-32]. The noise signal is input to the bistable or multistable nonlinear system. The system performance can be optimized by adjusting the parameters so that the effect of stochastic resonance achieves the best. The equation of the bistable Langevin system is as follows:

$$
\dot{x}=-\frac{\mathrm{d} U(x)}{\mathrm{d} x}+s(t)+n(t)
$$

where $U(x)$ is the potential function.

$$
U(x)=-\frac{1}{2} a x^{2}+\frac{1}{4} b x^{4},
$$

where $a$ and $b$ are system parameters. The potential function (8) has one unstable equilibrium point and two stable equilibrium points. The height of the potential barrier is $a^{2} /(4 b)$. $s(t)=\sum_{i=1}^{n} A_{i} \sin \left(2 \pi f_{i} t\right)$ represents an input signal with amplitude $A_{i}$ and frequency $f_{i} . n(t)=\sqrt{2 D} \varepsilon(t)$ represents the Gaussian white noise signal with intensity $D$, mean 0 , and variance 1 . The bistable Langevin system structure is shown in Figure 1.

The stochastic resonance problem is equivalent to a multidimensional-continuous optimization problem [33]. 


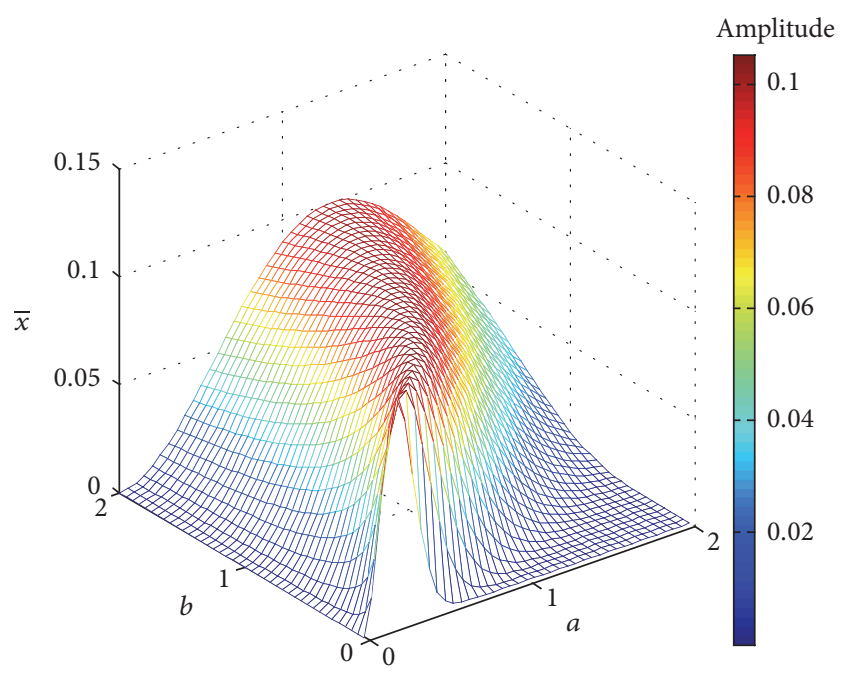

FIGURE 2: Influence of SR system parameters on amplitude.

The same set of input signals using different parameters of the system for stochastic resonance processing will produce different effect [34]. Setting $s(t)=A \sin \left(2 \pi f_{0} t\right)$, Figure 2 shows the response amplitude $\bar{x}$ changes with SR system parameters when $D=0.2, f_{0}=0.1 \mathrm{~Hz}$, and $A=0.1$. It is shown that there exist optimal system parameters $a, b$ to make the stochastic resonance performance optimal.

2.3. Quantum Particle Swarm Optimization. Quantum Particle Swarm Optimization was proposed on trajectory analysis of particle swarm optimization (PSO) and the quantum mechanism [35]. In the quantum space, the position and velocity of the particle cannot be determined simultaneously, so the wave function is used to describe the particle state [36]. The square of the wave function represents the probability of finding a particle at a particular position in the quantum space. By employing the Monte Carlo method, the position of a particle can be expressed as follows:

$$
x(t)=P \pm \frac{L}{2} \ln \left(\frac{1}{v}\right),
$$

where $v$ is a random number between 0 and $1 . L$ is the characteristic length of potential well which changes with the time as follows:

$$
L(t+1)=2 \beta\left|\sum_{i=1}^{N} \frac{P_{i}}{N}-x(t)\right|,
$$

where parameter $\beta$ is the contraction expansion coefficient and is used to control the convergence speed of the particle. $N$ is the number of particle swarms. $P_{i}$ is the local optimal value of particle $i$. Finally, the position of the particle can be expressed as follows:

$$
x(t+1)=P \pm \beta\left|\sum_{i=1}^{N} \frac{P_{\mathrm{i}}}{N}-x(t)\right| \ln \left(\frac{1}{v}\right) .
$$
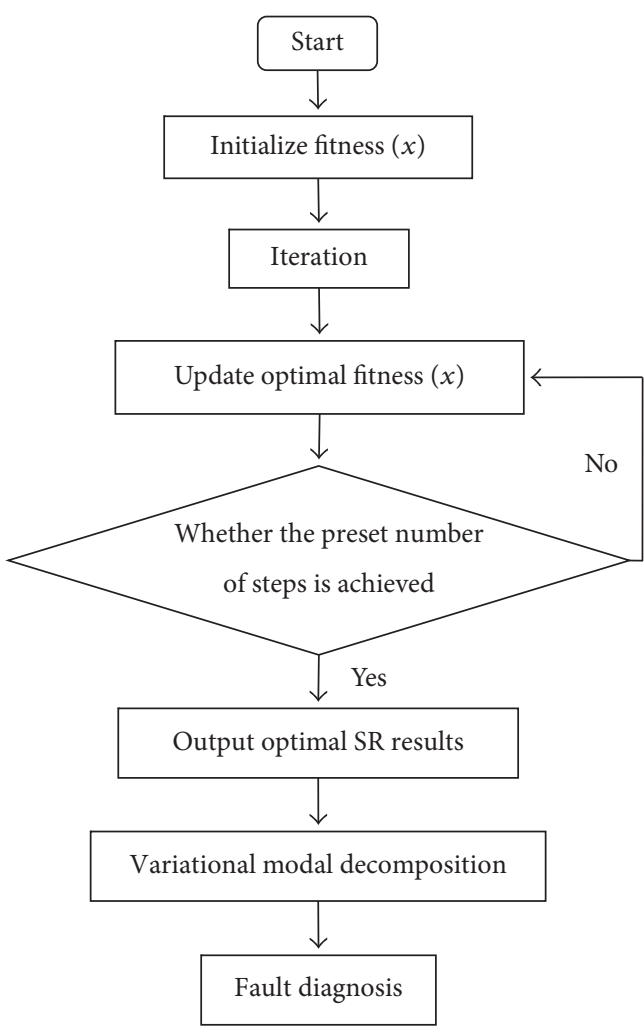

FIGURE 3: Flowchart of the proposed scheme.

\section{Proposed Fault Identification Scheme}

3.1. Fault Diagnosis Process. Different from the traditional fault identification method, this fault diagnosis process considers the noise feature of different input signal. In various moments, the fault features are varying degrees submerged in noise. Hence, fixed SR system parameters may be unable to suppress the noise and properly enhance the fault feature. This study proposes an adaptable and varying-scale stochastic resonance to overcome the shortcomings of the traditional method. The values of SR system parameters are optimized by using the quantum particle swarm optimization algorithm. The fitness of each particle is determined by the objective function. The performance of stochastic resonance system is mainly reflected in the signal to noise ratio (SNR) of the output signal [37]. Hence, the fitness function of QPSO algorithm is shown as follows:

$$
\text { fitness }(x)=10 \log \frac{P_{S}}{P_{N}},
$$

where $P_{S}:=2\left|X\left[k_{0}\right]\right|^{2}$ is the active power of the signal. $P_{N}:=$ $\sum_{k=0}^{L-1}|X[k]|^{2}-P_{S}$ is the active power of the noise. $|X[k]|$ is the discrete Fourier transform (DFT) of the sample sequence, which is defined according to the following formula:

$$
X[k]=\sum_{t=0}^{L-1} x_{t} \exp \left(-j \frac{2 \pi}{L} k t\right), \quad k=0,1, \ldots, L-1 .
$$

The procedures for this method of fault feature extraction are shown in Figure 3. 

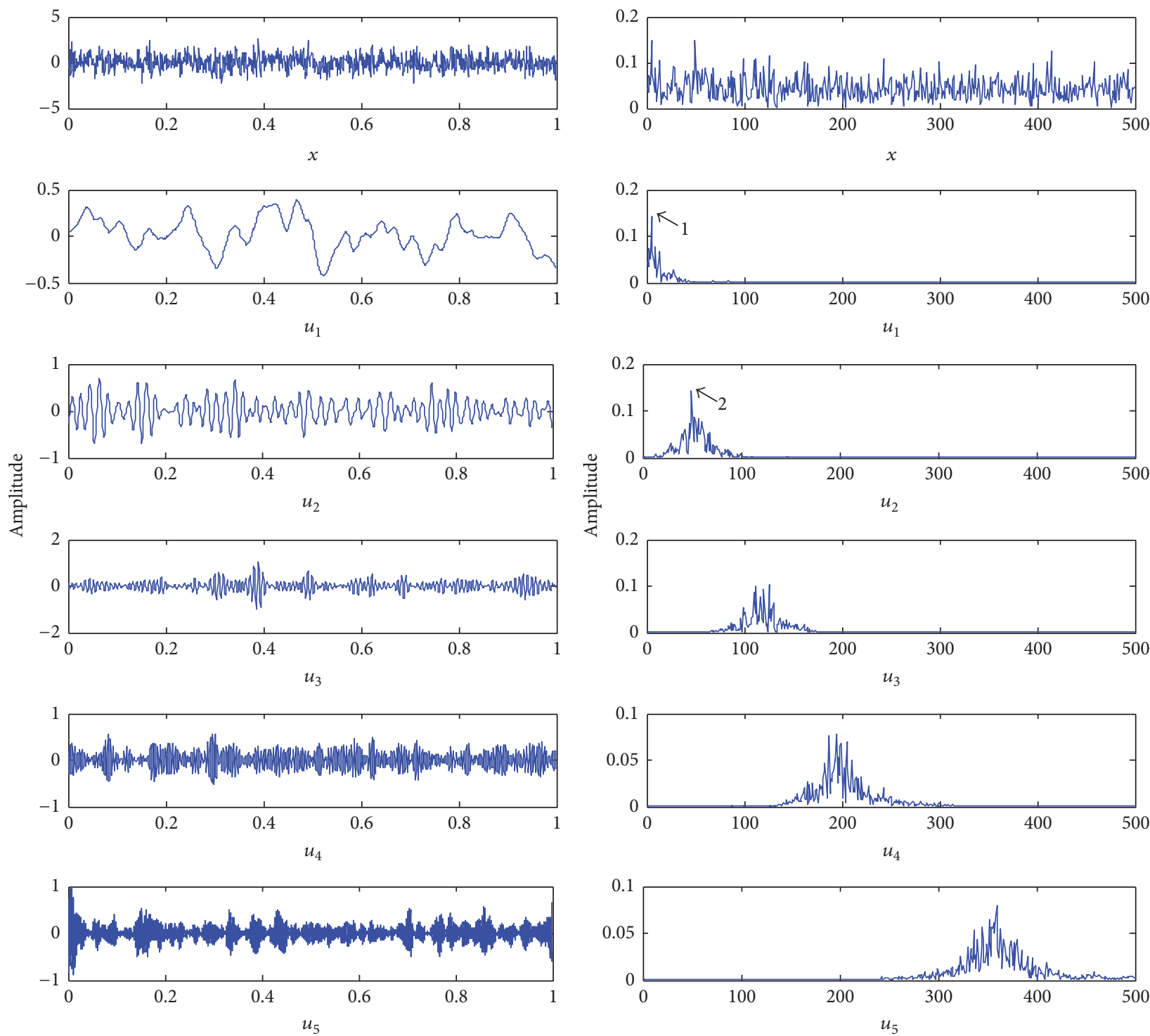

Time $t(\mathrm{~s})$

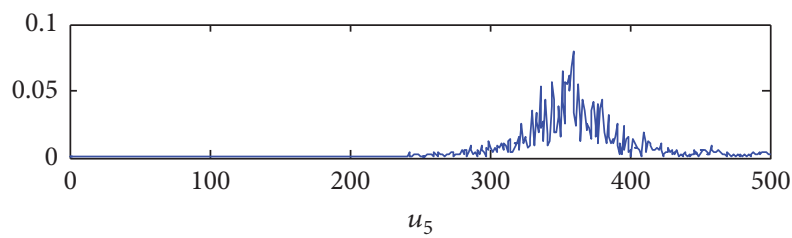

Frequency $f(\mathrm{~Hz})$

FIGURE 4: VMD analysis result of the simulated signal.

3.2. Simulation Signals Analysis. To demonstrate the differences between VMD and the improved VMD based on QPSO-SR, the synthesized signal which includes $5 \mathrm{~Hz}$ and $50 \mathrm{~Hz}$ sinusoidal signal and the Gauss white noise is used to simulate the fault signal. The analog signal expression is established as follows:

$$
\begin{aligned}
x(t)= & 0.15 \sin (2 \pi \times 5 t)+0.15 \sin (2 \pi \times 50 t) \\
& +n(t),
\end{aligned}
$$

where $t:=0.001: 0.001: 1$ is the sample time points. $n(t)$ is the Gauss white noise. The intensity of the Gauss white noise is 0.8 . For comparison, the simulated signal is first subjected to VMD decomposition. The quadratic penalty factor $\alpha$ selected the default value 2000 . The bandwidth $\tau$ selected the default value 0 . The number of variational mode components is determined by the method of observation center frequency [38]. Set the initial value of $K$ to 2 . The VMD is carried out with the number of variational mode components $K=$ $K+1$ and judged whether the center frequency of each mode component is close. When $K=6$ began to show the phenomenon of overdecomposition, the optimal mode number $K$ is 5 . The variational mode components can be obtained through VMD algorithm. The VMD results and the Fast Fourier Transform (FFT) are shown in Figure 4.

From Figure 4, it can be seen that the fault information cannot be identified from time spectrum and frequency spectrum of the simulated signal. After the VMD algorithm, the amplitude of $5 \mathrm{~Hz}$ (represented by point 1 ) in $u_{1}$ FFT is 0.1416 and the amplitude of $50 \mathrm{~Hz}$ (represented by point 2) in $u_{2}$ FFT is 0.1439 , showing the simulated fault information. And $u_{3}, u_{4}$, and $u_{5}$ show the noise signal information. 

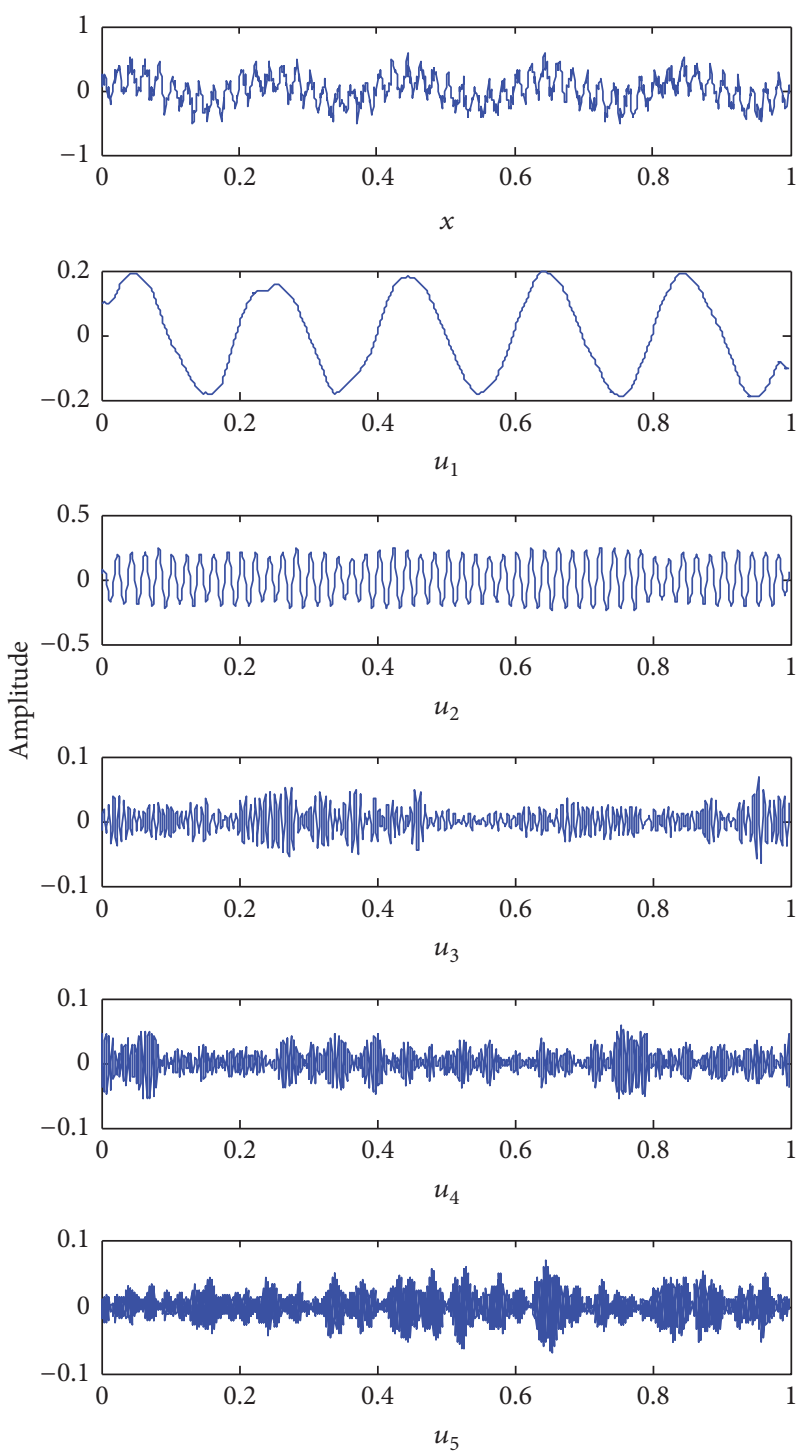

Time $t(\mathrm{~s})$
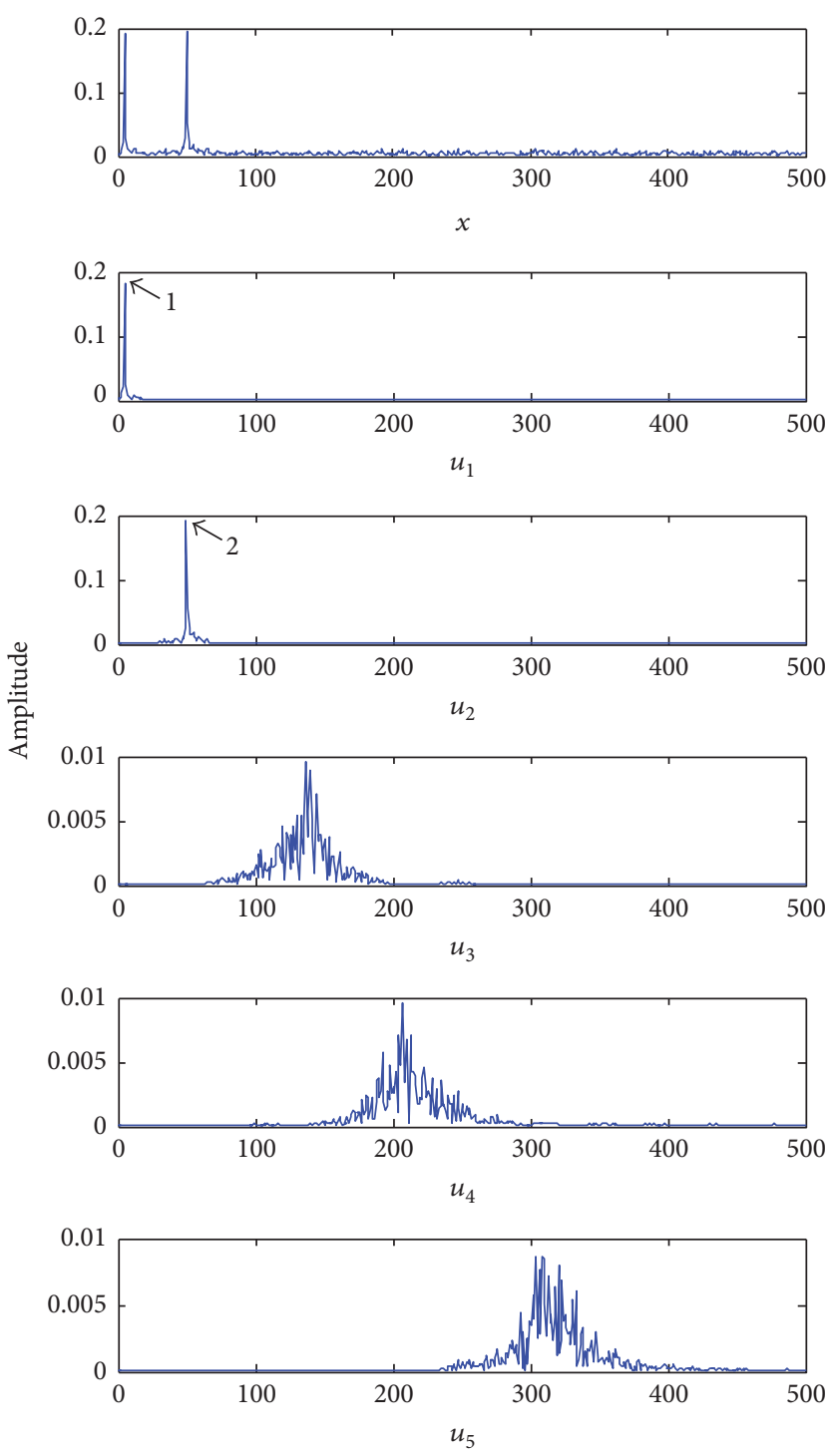

Frequency $f(\mathrm{~Hz})$

FIGURE 5: VMD analysis result of simulated signals based on QPSO-SR denoising.

However, $u_{1}, u_{2}$ mixed with the interference noise cannot accurately identify the simulated fault features.

In order to eliminate the effect of interference noise, the QPSO-SR method is used to denoise the simulated signal before VMD. The stochastic resonance system parameters a and $b$ are optimized by quantum particle swarm optimization algorithm. The optimization range of system parameters is from 0.01 to 2 . The optimization results show that SNR achieves the maximum value when $a=0.05$ and $b=0.17$. These system parameters are used to denoise the simulated signal. And then the signal processed by stochastic resonance is decomposed by VMD method. After QPSO-SR noise reduction, the result of VMD decomposition is shown in Figure 5.

From Figure 5, it can be seen that the interference noise in $u_{1}$ and $u_{2}$ is weakened. The amplitude of $5 \mathrm{~Hz}$ (represented by point 1) in $u_{1}$ FFT is increased from 0.1416 to 0.1820 .
The amplitude of $50 \mathrm{~Hz}$ (represented by point 2) in $u_{2}$ FFT is increased from 0.1439 to 0.1922 . The amplitude of the fault information frequency is obviously enhanced and the useful information is accurately identified. The amplitude of the mode components $u_{3}, u_{4}$, and $u_{5}$ which represent the noise signal is weakened. After the noise reduction by QPSO$\mathrm{SR}$, a part of the noise energy can be transmitted to the useful signal, which is helpful for VMD to obtain the useful information more accurately.

\section{Experimental Validations}

The bearing vibration signals are supplied by the Shenyang University of Technology. The rolling bearing vibration signals were measured by QPZZ-II rotating machinery vibration fault experimental platform. The experimental platform is shown in Figure 6. The motor rotational speed is at $1720 \mathrm{r} / \mathrm{min}$ 
TABLE 1: Geometry information of the tested rolling bearing.

\begin{tabular}{lcccc}
\hline $\begin{array}{l}\text { Inside diameter } \\
(\mathrm{mm})\end{array}$ & $\begin{array}{c}\text { Outside diameter } \\
(\mathrm{mm})\end{array}$ & $\begin{array}{c}\text { Bearing thickness } \\
(\mathrm{mm})\end{array}$ & $\begin{array}{c}\text { Ball diameter } \\
(\mathrm{mm})\end{array}$ & $\begin{array}{c}\text { Pitch diameter } \\
(\mathrm{mm})\end{array}$ \\
\hline 25.00 & 52.00 & 15.00 & 7.94 & 39.04 \\
\hline
\end{tabular}

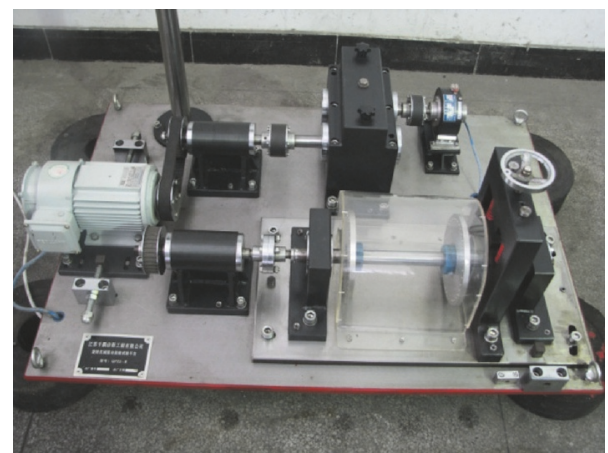

FIGURE 6: Experimental facility.

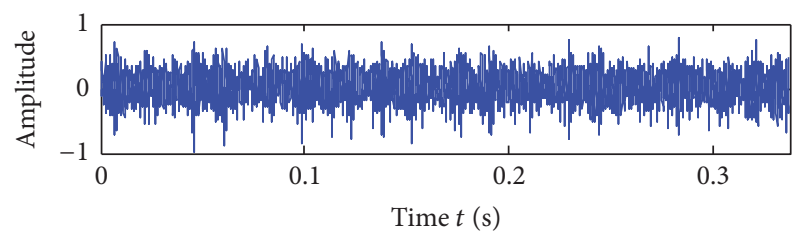

(a)

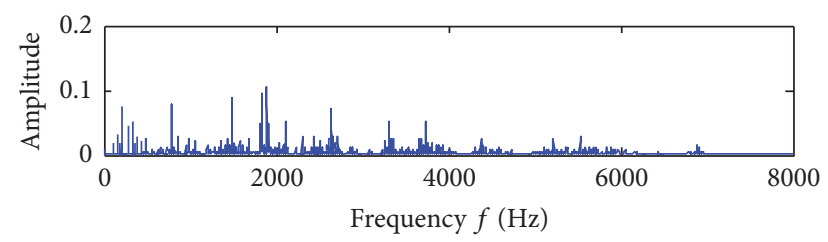

(b)

FIgURE 7: Raw vibration signal.

to $1797 \mathrm{r} / \mathrm{min}$. The digital data used in this analysis were collected at 16,000 samples per second and the sampling number is 5468 . A $0.2 \mathrm{~mm}$ wide and $0.1 \mathrm{~mm}$ deep groove was wire-cut on the surface of the bearing inner ring to simulate early fault. The detailed information of the tested rolling bearing is presented in Table 1. The fault feature frequency of the tested rolling bearing is presented in Table 2 .

The time domain spectrum and frequency domain spectrum of rolling bearing vibration signal with inner ring fault are shown in Figure 7. The SNR of raw vibration signal is $-15.3097 \mathrm{~dB}$. It can be seen from the waveform that there are obvious interference noise and shock in the rolling bearing vibration signal and the frequency component is rich. Therefore, the fault information cannot be obtained from the spectrum directly.

The raw vibration signal is decomposed by VMD. The decomposition which is obtained by VMD is processed by
TABLE 2: Fault feature frequency of the tested rolling bearing.

\begin{tabular}{lccc}
\hline $\begin{array}{l}\text { Inner ring } \\
(\mathrm{Hz})\end{array}$ & $\begin{array}{c}\text { Outer ring } \\
(\mathrm{Hz})\end{array}$ & $\begin{array}{c}\text { Rolling element } \\
(\mathrm{Hz})\end{array}$ & $\begin{array}{c}\text { Cage element } \\
(\mathrm{Hz})\end{array}$ \\
\hline $5.4152 f_{r}$ & $3.5848 f_{r}$ & $4.7135 f_{r}$ & $0.39828 f_{r}$ \\
\hline$f_{r}=29.95 \mathrm{~Hz}$. & & &
\end{tabular}

TABLE 3: The SNR of the output signal.

\begin{tabular}{lcc}
\hline$a$ & $b$ & $\mathrm{SNR}_{\text {out }}(\mathrm{dB})$ \\
\hline 0.01 & 0.07 & 3.8942 \\
0.03 & 0.06 & 1.8319 \\
0.05 & 0.16 & 3.0896 \\
0.10 & 0.12 & 2.5896 \\
1.19 & 1.97 & 5.0046 \\
\hline
\end{tabular}

using Envelope analysis and fast Fourier transform algorithm. The VMD results and the FFT are shown in Figure 8. In contrast, replace VMD with EEMD and perform the same processing on the raw signal. The EEMD results and the FFT are shown in Figure 9. The EEMD decomposition result reveals that the phenomena of mode mixing make the early fault indistinguishable. The VMD decomposition result reveals that the VMD method solves the phenomena of mode mixing but the component still contains noise disturbance and shock. VMD algorithm has not realized the potential that was expected of it. Characteristic frequency $162 \mathrm{~Hz}$ (represented by point 1), double frequency $324 \mathrm{~Hz}$ (represented by point 2), and third frequency $486 \mathrm{~Hz}$ (represented by point 3 ) of bearing inner ring fault are not obvious in the decomposition result (see Figure 8). This shows that it is difficult to ensure the accuracy of the fault diagnosis in the practical engineering if we use the VMD algorithm independently.

The traditional stochastic resonance method is employed to process the raw vibration signal. SR system parameters are selected according to experience. The parameter $a$ takes on $0.01,0.03,0.05,0.10$, and 1.19 , respectively. The parameter $b$ takes on $0.07,0.06,0.16,0.12$, and 1.97 , respectively. The raw vibration signal is subjected to SR processing by the above five sets of parameters, respectively. The SNR of the output signal is shown in Table 3.

The proposed fault weak signal extraction method based on VMD and QPSO-SR denoising is employed to process the raw vibration signal. Firstly, the raw signal is preprocessed with QPSO-SR method. According to the raw signal characteristics, the quantum particle swarm optimization algorithm is used to optimize the SR system parameters. The signal is 

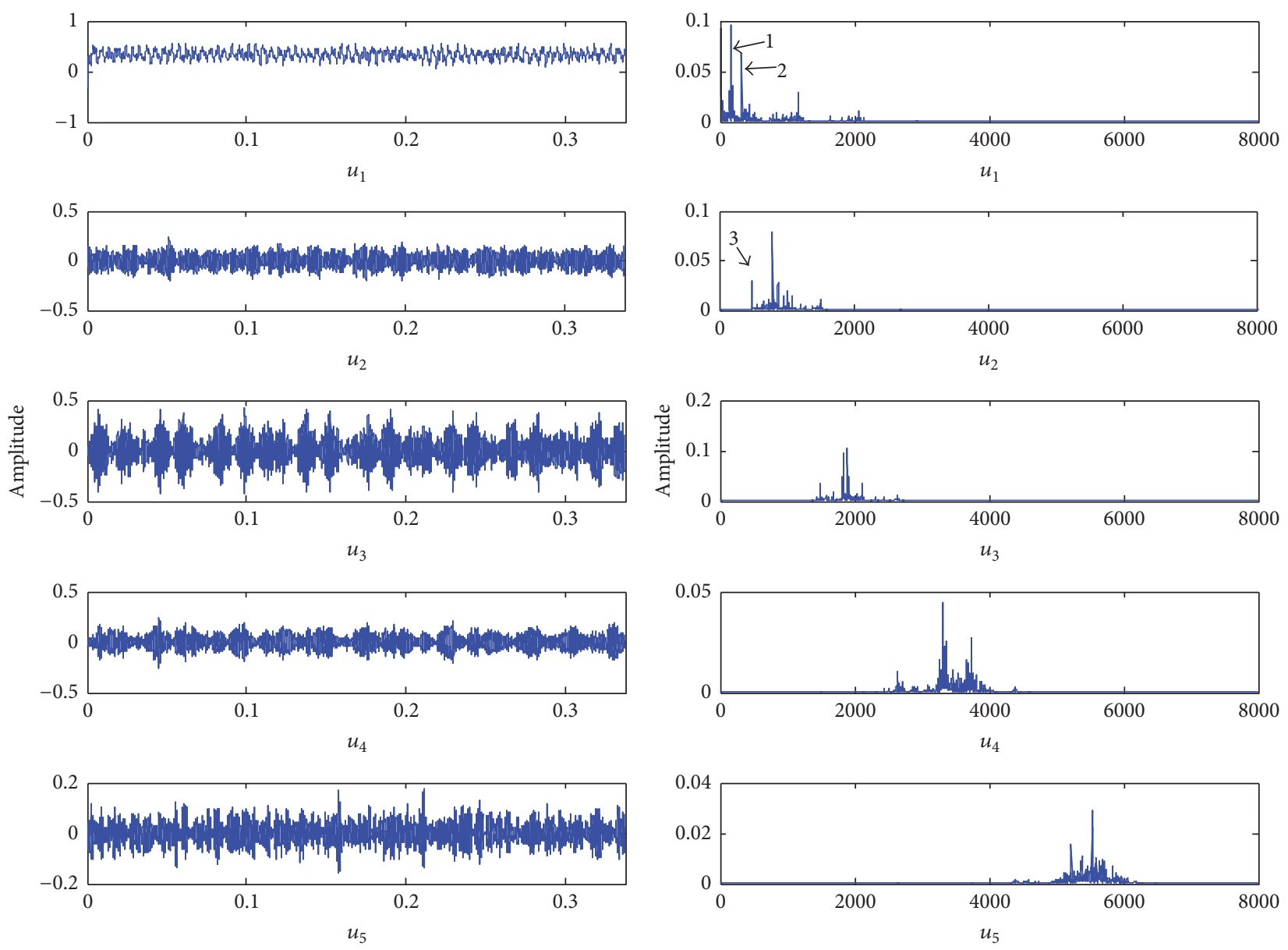

Time $t(\mathrm{~s})$

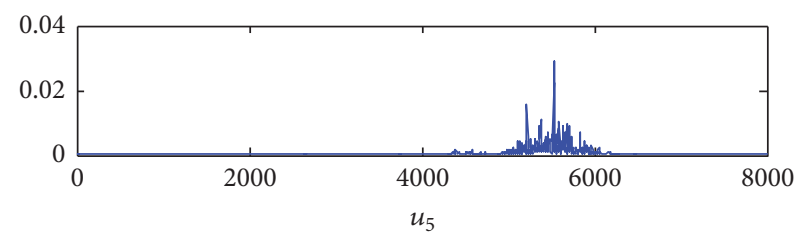

Frequency $f(\mathrm{~Hz})$

FIGURE 8: VMD analysis result of the rolling bearing vibration signal.

subjected to SR processing based on the optimal parameters. The SNR of the output signal is $7.6465 \mathrm{~dB}$. Evidently, the processed signals have been well denoised with the aid of QPSO-SR method. The frequency domain information exchange method is used to overcome the limitation of SR to large parameter signal. Liu et al. [39] described the specific calculation process of the frequency domain information exchange method in detail. The signal is subjected to VMD after denoising. Center frequency corresponding to different $K$ is shown in Table 4 . When $K$ is 6 , overdecomposition began to happen, so $K$ was chosen as 5 . The time domain and frequency domain waveforms of the results are shown in Figure 10.

From Figure 10, it can be seen that the VMD results are less affected by interference noise. Characteristic frequency (represented by point 1), double frequency (represented by point 2), and third frequency (represented by point 3 ) of bearing inner ring fault are obvious in the result. The fault feature is highlighted and the noise component is weakened. The bearing fault type can be analyzed intuitively and effectively. Compared with the original signal which has not been processed by QPSO-SR method, the shock
TABLE 4: Center frequency corresponding to different $K$.

\begin{tabular}{ccccccc}
\hline$K$ & \multicolumn{5}{c}{ Center frequency $(\mathrm{Hz})$} \\
\hline 2 & 1767 & 4784 & - & - & - & - \\
3 & 1767 & 2789 & 4784 & - & - & - \\
4 & 824 & 1767 & 2789 & 4784 & - & - \\
5 & 824 & 1767 & 2789 & 3641 & 4784 & - \\
6 & 824 & 1767 & 2789 & 3641 & 4668 & 4784 \\
\hline
\end{tabular}

wave of the processed signal is obviously reduced in time domain waveform. The energy distribution of the processed signal spectrum is also more concentrated in the bearing fault frequency band. This proofs that a portion of the noise energy can be passed to the useful signal by the appropriate SR method, which is helpful for VMD to obtain the useful information more accurately.

\section{Conclusion}

This study presents a novel weak fault extraction method. This method includes QPSO-SR method and variational 

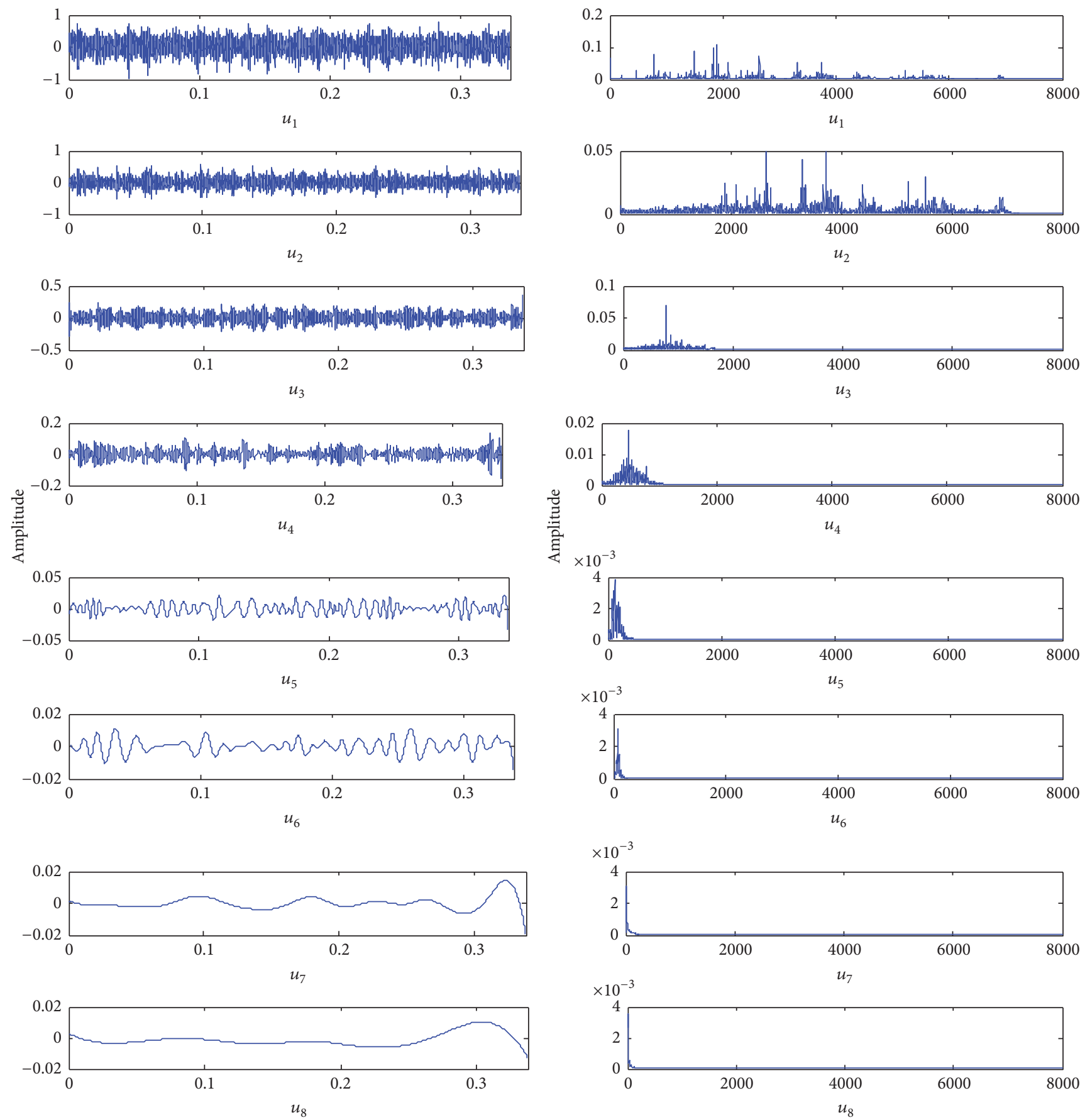

Time $t(\mathrm{~s})$

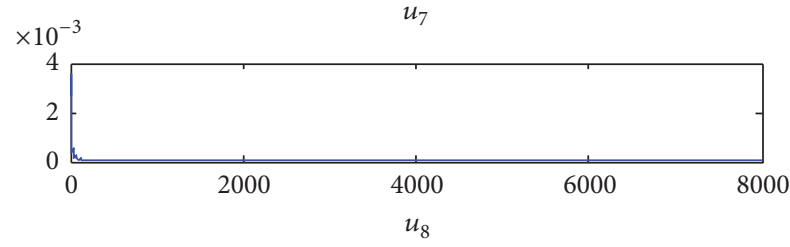

Frequency $f(\mathrm{~Hz})$

FIGURE 9: EEMD analysis result of the rolling bearing vibration signal.

mode decomposition. QPSO-SR method is an adaptive denoising method that can transmit a part of the noise energy to the weak fault signal. First, QPSO-SR method is proposed to enhance the fault characteristic signal and reduce the influence from the interference noise and the shock. According to the characteristics of the original fault vibration signal, the parameters of SR system are optimized by QPSO algorithm dynamically. Thereafter, the optimal SR system is used for denosing and the processed signal is decomposed by VMD method. The failure feature information can be extracted through the FFT of the VMD component. The results obtained in the investigation of the simulated fault signal and the rolling bearing data set show that the proposed scheme avoids the influence of the interference noise and 

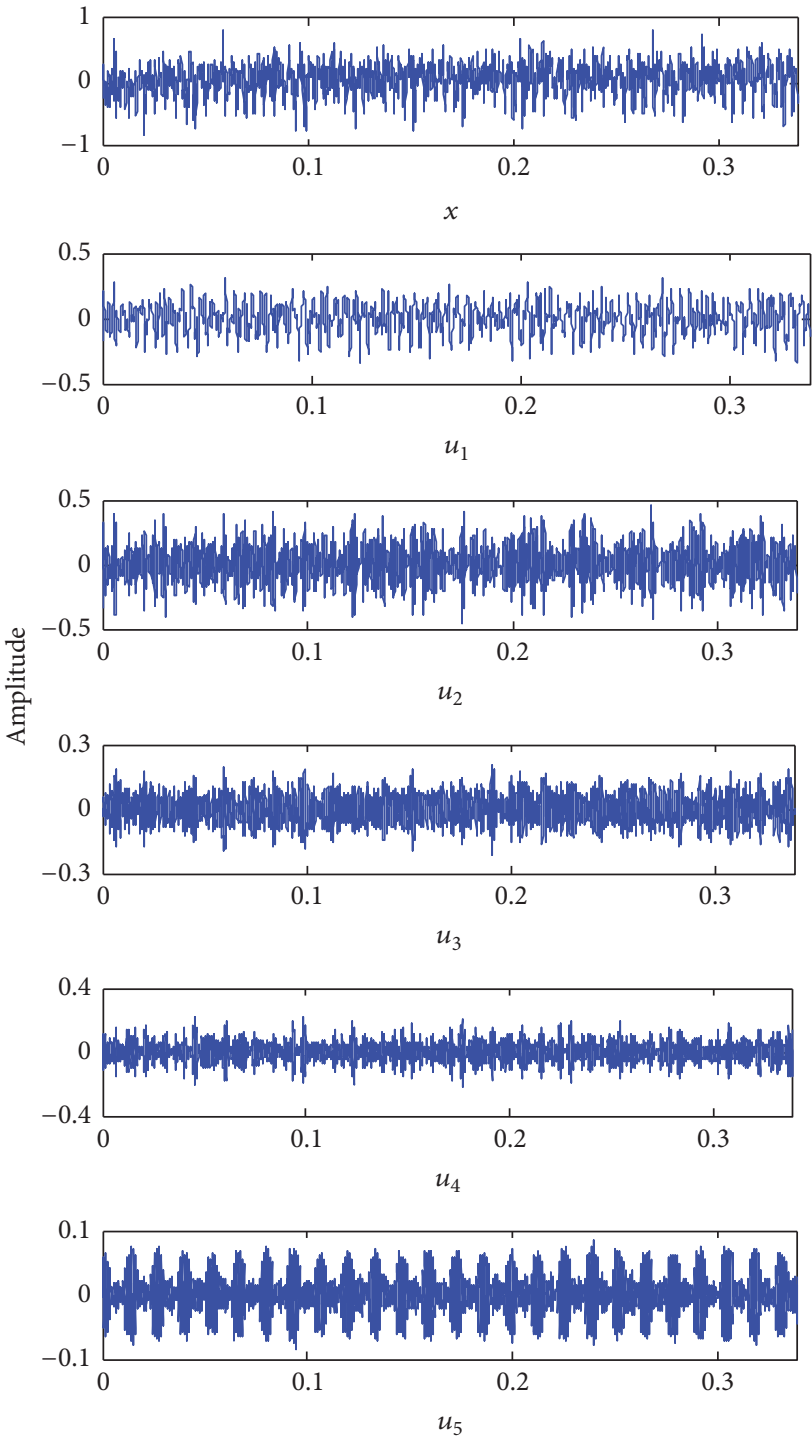

Time $t(\mathrm{~s})$
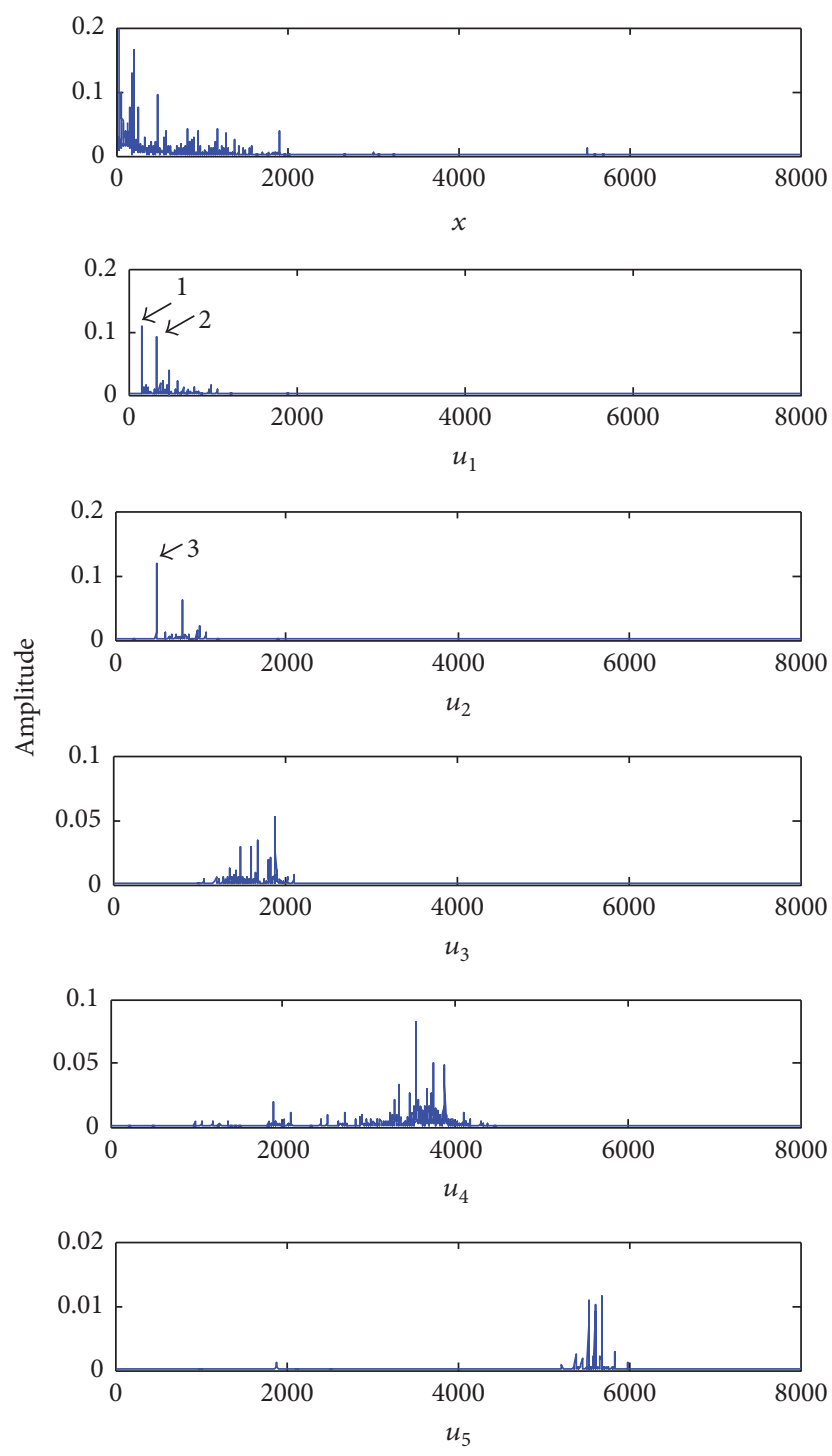

Frequency $f(\mathrm{~Hz})$

FIGURE 10: VMD analysis result of the rolling bearing vibration signal based on QPSO-SR denoising.

the shock. The proposed scheme reaches a higher degree of accuracy in machinery fault diagnosis compared with previous VMD method.

\section{Conflicts of Interest}

The authors declare that they have no conflicts of interest.

\section{Acknowledgments}

This research was financially supported by the National Natural Science Foundation of China (Grants nos. 51675350 and 51575361) and the Doctoral Research Initial Fund of Liaoning Province (201601154).

\section{References}

[1] J. Shuai, C. Shen, and Z. Zhu, "Adaptive Morphological Feature Extraction and Support Vector Regressive Classification for Bearing Fault Diagnosis," International Journal of Rotating Machinery, vol. 2017, Article ID 2384184, 2017.

[2] C. Peeters, P. Guillaume, and J. Helsen, "A comparison of cepstral editing methods as signal pre-processing techniques for vibration-based bearing fault detection," Mechanical Systems and Signal Processing, vol. 91, pp. 354-381, 2017.

[3] L. Barbini, A. P. Ompusunggu, A. J. Hillis, J. L. du Bois, and A. Bartic, "Phase editing as a signal pre-processing step for automated bearing fault detection," Mechanical Systems and Signal Processing, vol. 91, pp. 407-421, 2017.

[4] A. Kumar and R. Kumar, "Least Square Fitting for Adaptive Wavelet Generation and Automatic Prediction of Defect Size 
in the Bearing Using Levenberg-Marquardt Backpropagation," Journal of Nondestructive Evaluation, vol. 36, no. 1, article no. 7, 2017.

[5] D. P. Jena and S. N. Panigrahi, "Automatic gear and bearing fault localization using vibration and acoustic signals," Applied Acoustics, vol. 98, pp. 20-33, 2015.

[6] L. L. Xia, L. Wen, H. M. Liu, Y. Chu, and J. J. Tai, "Fault diagnosis and pattern recognition of power transformer based on improved PSO-BPNN," Journal ofShenyang University of Technology, vol. 38, no. 6, pp. 606-611, 2016.

[7] C. Mishra, A. K. Samantaray, and G. Chakraborty, "Bond graph modeling and experimental verification of a novel scheme for fault diagnosis of rolling element bearings in special operating conditions," Journal of Sound and Vibration, vol. 377, pp. 302330, 2016.

[8] L. J. Meng and J. W. Xiang, "Rolling bearing fault diagnosis based on second generation wavelet denoising and improved EEMD," Applied Mechanics and Materials, vol. 556-562, pp. 2677-2680, 2014.

[9] C. Yi, Y. Lv, Z. Dang, H. Xiao, and X. Yu, "Quaternion singular spectrum analysis using convex optimization and its application to fault diagnosis of rolling bearing," Measurement, vol. 103, pp. 321-332, 2017.

[10] L. Xie, X. Lang, J. Chen, A. Horch, and H. Su, "Timevarying oscillation detector based on improved LMD and robust Lempel-Ziv complexity," Control Engineering Practice, vol. 51, pp. 48-57, 2016.

[11] C. Yi, Y. Lv, M. Ge, H. Xiao, and X. Yu, “Tensor singular spectrum decomposition algorithm based on permutation entropy for rolling bearing fault diagnosis," Entropy, vol. 19, no. 4, article no. 139, 2017.

[12] K. Dragomiretskiy and D. Zosso, "Variational mode decomposition," IEEE Transactions on Signal Processing, vol. 62, no. 3, pp. 531-544, 2014.

[13] C. Yi, Y. Lv, and Z. Dang, "A fault diagnosis scheme for rolling bearing based on particle swarm optimization in variational mode decomposition," Shock and Vibration, vol. 2016, Article ID 9372691, 10 pages, 2016.

[14] T. Dutta, U. Satija, B. Ramkumar, and M. S. Manikandan, "A novel method for automatic modulation classification under non-Gaussian noise based on variational mode decomposition," in Proceedings of the 22nd National Conference on Communication, NCC 2016, ind, March 2016.

[15] Y. J. W. Ngouongo, G. D. Kenmoe, and T. C. Kofane, "Effect of coupling on stochastic resonance and stochastic antiresonance processes in a unidirectionally $\mathrm{N}$-coupled systems in periodic sinusoidal potential," Physica A: Statistical Mechanics and its Applications, vol. 472, pp. 25-31, 2017.

[16] Z.-H. Lai and Y.-G. Leng, "Weak-signal detection based on the stochastic resonance of bistable Duffing oscillator and its application in incipient fault diagnosis," Mechanical Systems and Signal Processing, vol. 81, pp. 60-74, 2016.

[17] D. Han, P. li, S. An, and P. Shi, "Multi-frequency weak signal detection based on wavelet transform and parameter compensation band-pass multi-stable stochastic resonance," Mechanical Systems and Signal Processing, vol. 70-71, pp. 995-1010, 2016.

[18] A. Rother, M. Jelali, and D. Söffker, "A brief review and a first application of time-frequency-based analysis methods for monitoring of strip rolling mills," Journal of Process Control, vol. 35, pp. 65-79, 2015.

[19] L. Barbini, M. O. T. Cole, A. J. Hillis, and J. L. Du Bois, "Weak signal detection based on two dimensional stochastic resonance," in Proceedings of the 23rd European Signal Processing Conference, EUSIPCO 2015, pp. 2147-2151, Nice, Franc, September 2015.

[20] R. Castiglione, L. Garibaldi, S. Marchesiello, and R. Serra, "Stochastic Resonance algorithms to enhance damage detection in bearing faults," Matec Web of Conferences, vol. 20, p. 07005, 2015.

[21] S. L. Gao, S. C. Zhong, K. Wei, and H. Ma, "Weak singal detection based on chaos and stochastic resonance," Acta Physica Sinica, vol. 61, no. 18, Article ID 180501, 2012.

[22] S. Lu, Q. He, H. Zhang, and F. Kong, "Rotating machine fault diagnosis through enhanced stochastic resonance by full-wave signal construction," Mechanical Systems and Signal Processing, vol. 85, pp. 82-97, 2017.

[23] L.-f. Lin, L. Yu, H. Wang, and S. Zhong, "Parameter-adjusted stochastic resonance system for the aperiodic echo chirp signal in optimal FrFT domain," Communications in Nonlinear Science and Numerical Simulation, vol. 43, pp. 171-181, 2017.

[24] B. J. Breen, J. G. Rix, S. J. Ross et al., "Harvesting wind energy to detect weak signals using mechanical stochastic resonance," Physical Review E: Statistical, Nonlinear, and Soft Matter Physics, vol. 94, no. 6, Article ID 062205, 2016.

[25] P. Shi, C. Su, and D. Han, "Fault diagnosis of rotating machinery based on adaptive stochastic resonance and AMD-EEMD," Shock and Vibration, vol. 2016, Article ID 9278581, 11 pages, 2016.

[26] J. Xiang and Y. Zhong, "A two-step method using Duffing oscillator and stochastic resonance to detect mechanical faults," in Proceedings of the 2016 IEEE International Instrumentation and Measurement Technology Conference, I2MTC 2016, Taipei, Taiwan, May 2016.

[27] W.-X. Liu, Y.-J. Wang, X. Liu, and M.-J. Zhang, "Weak thruster fault detection for AUV based on stochastic resonance and wavelet reconstruction," Journal of Central South University, vol. 23, no. 11, pp. 2883-2895, 2016.

[28] Z. Li, J. Chen, Y. Zi, and J. Pan, "Independence-oriented VMD to identify fault feature for wheel set bearing fault diagnosis of high speed locomotive," Mechanical Systems and Signal Processing, vol. 85, pp. 512-529, 2017.

[29] R. Benzi, A. Sutera, and A. Vulpiani, "The mechanism of stochastic resonance," Journal of Physics A: Mathematical and General, vol. 14, no. 11, pp. L453-L457, 1981.

[30] H. Zhang, Y. Zheng, and F. Kong, "Weak impulsive signals detection based on step-varying asymmetric stochastic resonance," Proceedings of the Institution of Mechanical Engineers, Part C: Journal of Mechanical Engineering Science, vol. 231, no. 2, pp. 242-262, 2017.

[31] M. Uzuntarla, J. J. Torres, P. So, M. Ozer, and E. Barreto, "Double inverse stochastic resonance with dynamic synapses," Physical Review E: Statistical, Nonlinear, and Soft Matter Physics, vol. 95, no. 1, Article ID 012404, 2017.

[32] P. Zhou, S. Lu, F. Liu, Y. Liu, G. Li, and J. Zhao, "Novel synthetic index-based adaptive stochastic resonance method and its application in bearing fault diagnosis," Journal of Sound and Vibration, vol. 391, pp. 194-210, 2017.

[33] P. Addesso, V. Pierro, and G. Filatrella, "Interplay between detection strategies and stochastic resonance properties," Communications in Nonlinear Science and Numerical Simulation, vol. 30, no. 1-3, pp. 15-31, 2016.

[34] Z. Qiao, Y. Lei, J. Lin, and F. Jia, "An adaptive unsaturated bistable stochastic resonance method and its application in 
mechanical fault diagnosis," Mechanical Systems and Signal Processing, vol. 84, pp. 731-746, 2017.

[35] . Shuyuan Yang, . Min Wang, and . Licheng jiao, "A quantum particle swarm optimization," in Proceedings of the 2004 Congress on Evolutionary Computation, pp. 320-324, Portland, OR, USA.

[36] W. Fang, J. Sun, Y. Ding, X. Wu, and W. Xu, "A review of quantum-behaved particle swarm optimization," IETE Technical Review, vol. 27, no. 4, pp. 336-347, 2010.

[37] D. Yang, H. Li, Y. Hu, J. Zhao, H. Xiao, and Y. Lan, "Vibration condition monitoring system for wind turbine bearings based on noise suppression with multi-point data fusion," Journal of Renewable Energy, vol. 92, pp. 104-116, 2016.

[38] A. A. Abdoos, "A new intelligent method based on combination of VMD and ELM for short term wind power forecasting," Neurocomputing, vol. 203, pp. 111-120, 2016.

[39] J. J. Liu, Y. G. Leng, Z. H Lai, and T. Dan, "Stochastic resonance based on frequency domain information exchange," Acta Physica Sinica, vol. 65, no. 22, Article ID 220501, 2016. 


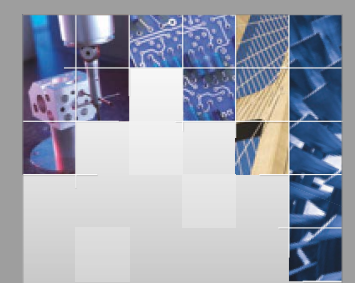

\section{Enfincering}
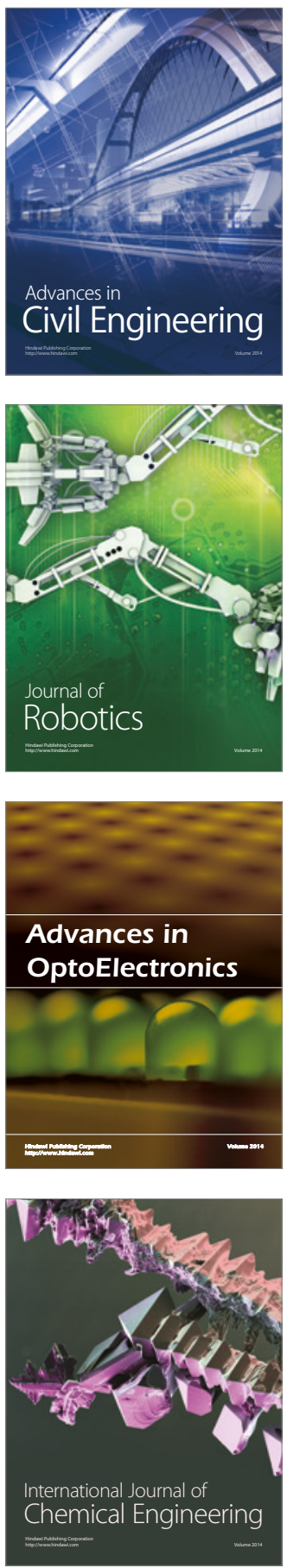

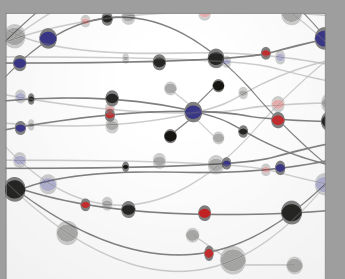

The Scientific World Journal

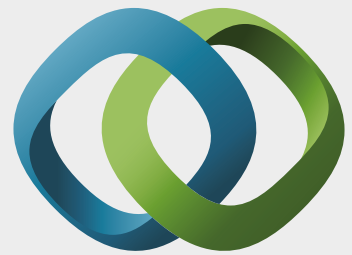

\section{Hindawi}

Submit your manuscripts at

https://www.hindawi.com
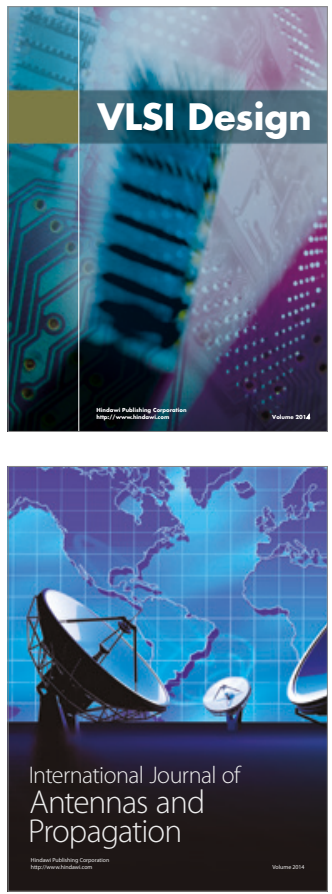

\section{Rotating}

Machinery
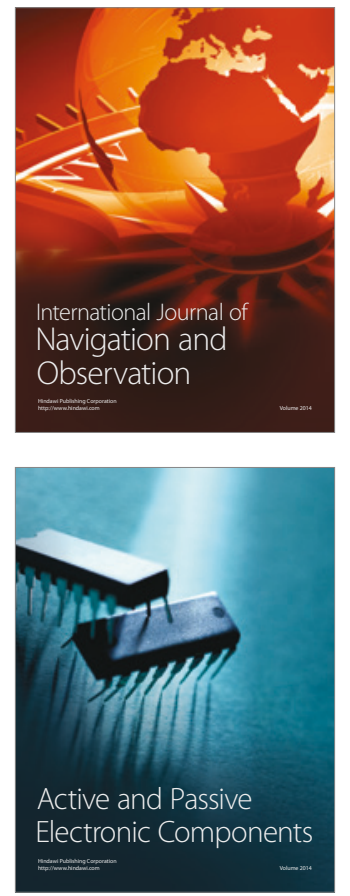
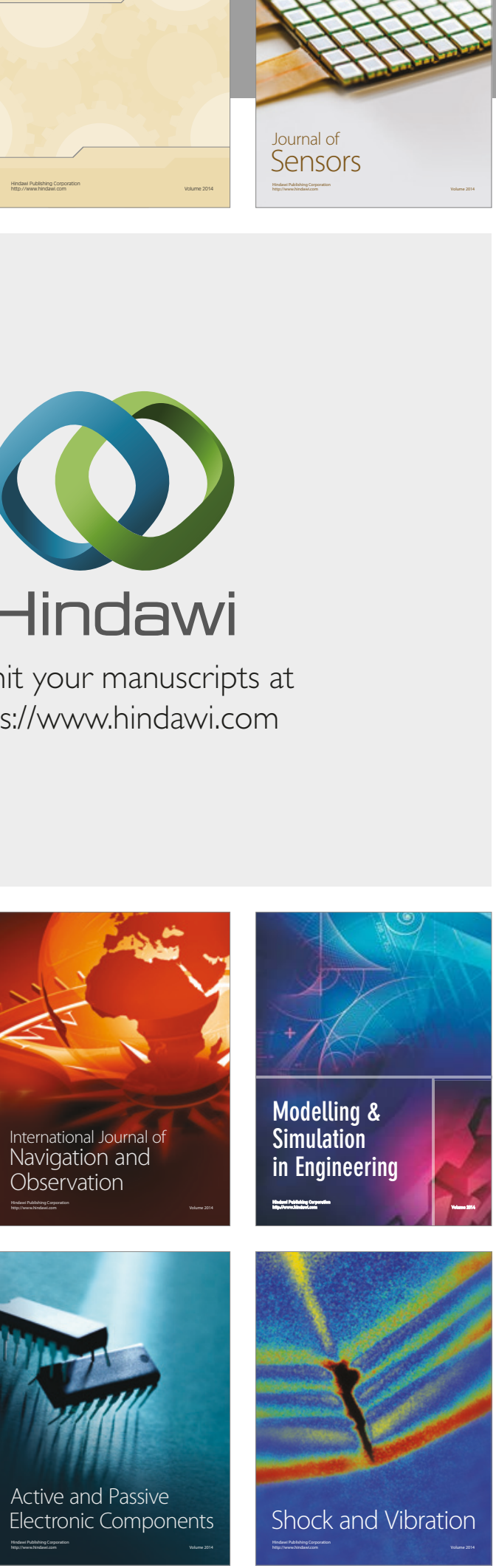
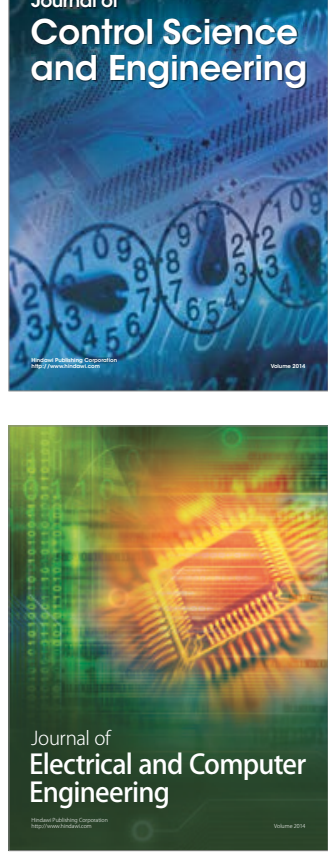

Distributed

Journal of

Control Science

and Engineering
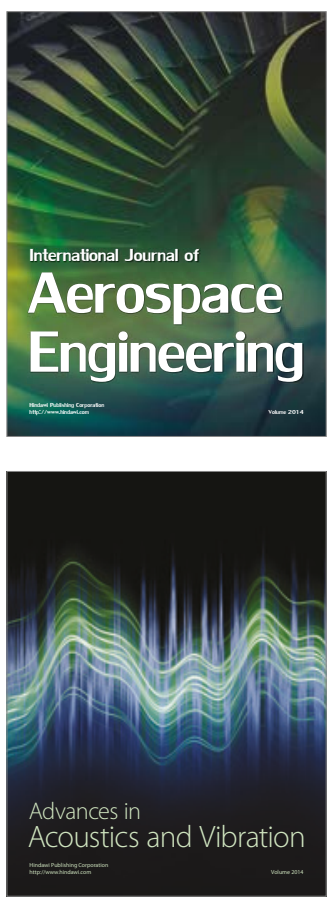

Sensor Networks 